\title{
Gene expression profiling of oxidative stress on atrial fibrillation in humans
}

\author{
Young Hoon $\mathrm{Kim}^{1 *}$, Ji Hye Lee ${ }^{2 *}$ \\ Do Sun Lim ${ }^{1}$, Wan Joo Shim ${ }^{1}$ \\ Young Moo Ro ${ }^{1}$, Gil Hong Park ${ }^{2}$ \\ Kevin G. Becker ${ }^{3}$, Yoon S. Cho-Chung ${ }^{4}$ \\ and Meyoung-kon $\mathrm{Kim}^{2,5}$ \\ ${ }^{1}$ Department of Internal Medicine \\ ${ }^{2}$ Department of Biochemistry \\ College of Medicine, Korea University \\ Seoul, 136-701 Korea \\ ${ }^{3}$ DNA Array Unit, National Institute on Aging \\ National Institutes of Health \\ Baltimore, MD 21224-6820 \\ ${ }^{4}$ Cellular Biochemistry Section \\ Basic Research Laboratory, CCR \\ National Cancer Institute \\ National Institutes of Health \\ Bethesda, MD 20892-1750 \\ ${ }^{5}$ Corresponding Author: Tel, 82-2-920-6184; \\ Fax, 82-2-923-0480; E-mail, jerrykim@korea.ac.kr \\ *The first two authors contributed equally to this work.
}

Accepted 24 July 2003

Abbreviations: AF, atrial fibrillation; cDNA, complementary deoxyribonucleic acid; $\mathrm{O}_{2}^{-}$, superoxide anion; ROS, reactive oxygen species

\section{Abstract}

Atrial Fibrillation (AF) is thought be caused by oxidative stress. Oxidative stress at the cellular level results from many factors, including exposure to alcohol, medications, cold, toxins or radiation. In this study we investigated gene transcriptional profiles on the human myocardial tissues from $A F$ and oxidative stress conditions. Right atrial appendages were obtained from $A F$ patients $(n=26)$ undergoing the Maze procedure, and from control patients $(n=26)$ who were in normal sinus rhythm and undergoing coronary artery bypass graft operation. To examine the effects of oxidative stress on $A F$, we used radioactive complementary DNA (cDNA) microarrays to evaluate changes in the expression of 1,152 known genes. This technology, which monitors thousands of genes simultaneously, gives us a better picture of the interactions between AF and oxidative stress. Total RNAs prepared from the retrieved tissues were used to synthesize ${ }^{33} \mathrm{P}$-labeled cDNAs by reverse transcription and hybridized to cDNA microarrays. Gene expression profiles showed that 30 genes were upregulated and 25 were downregulated in AF patients compared with control patients. Moreover, comparison rank analysis revealed that the expression of five genes related to reactive oxygen species (ROS)-including flavin containing monooxygenase 1 , monoamine oxidase $B$, ubiquitin specific protease 8 , tyrosinase-related protein 1 , and tyrosine 3-monooxygenase-increased by more than 2.0 of the Z-ratio, and two genes related to antioxidantsincluding glutathione peroxidase 1 , and heme oxygenase 2-decreased to the Z-ratio levels of $\leq-2.0$. Apparently, a balanced regulation of pro- and anti-oxidation can be shifted toward pro-oxidation and can result in serious damage similar to that of human AF. Western blotting analysis confirmed the upregulation of tyrosinase-related protein 1 and tyrosine 3-monooxygenase and the downregulation of heme oxygenase 2 . These results suggested that the gene expression pattern of myocardial tissues in AF patients can be associated with oxidative stress, resulting in a significant increase in ROS. Thus, the cDNA microarray technique was useful for investigating transcription profiles in AF. It showed that the intracellular mechanism of oxidative stress plays a pivotal role in the pathologic progression of $A F$ and offers novel insight into potential treatment with antioxidants.

Keywords: atrial fibrillation; cDNA microarray; gene expression profile; oxidative stress

\section{Introduction}

Atrial fibrillation $(A F)$, the most prevalent sustained arrhythmia, affects more than 900,000 Koreans and more than 5 million people worldwide. Its incidence increases with age and it is present in structural heart disease (Kopecky et al., 1987). The prevalence of AF increases strikingly with advancing age; it occurs in approximately $2 \%$ of men $(2.2 \%)$ and women $(1.7 \%)$ older than age 20 , and $5 \%$ of those older than age 
65 (Prystowsky et al., 1996). Recent data suggest that AF-related hospital stays are markedly greater than for any other arrhythmia. Nevertheless, information about its incidence and prevalence in a general population is sparse.

AF-associated morbidity is related to excessive ventricular rate and systemic embolization, and may cause syncope, fatigue, or cardiomyopathy. AF patients have marked atrial dilatation and atrial myocyte hypertrophy with increased interstitial fibrosis and fatty deposition. It may be seen in normal subjects, particularly during emotional stress or following surgery, exercise, or acute alcoholic intoxication (Mihm et al., 2001). AF is a frequent postoperative complication of cardiac surgery, with a reported $20 \%$ to $50 \%$ incidence, increasing the risk of stroke. Patients undergoing coronary atrial bypass graft surgery have increased plasma lipid peroxidation and decreased cardiac glutathione levels following release of cross clamp, and these changes persist for at least $24 \mathrm{~h}$ after cardiac surgery. Similarly, increased free-radical production in canine heart subjects lead to rapid ventricular pacing, and antioxidants can improve cardiac function in animals with pacing-induced failure (Carnes et al., 2001). Free radical is formed via the diffusion rate-limited reaction of nitric oxide and superoxide anion, and is known to oxidize cellular lipids, proteins, and DNA, and to promote cardiac cell death via necrosis and/or apoptosis.

In this study, we assessed a causal relationship between reactive oxygen species (ROS) and AF. Because any oxygen-involving free radical can be referred to as ROS (Goldfarb, 1999). ROS play important roles in many cardiovascular pathologies, including atrial fibrillation, atherosclerosis, and others. These molecules are so reactive that they act in situ very close to where they are generated. Therefore, most cell structures-including membranes, structural proteins, enzymes, and nucleic acids-are vulnerable and can become targets for mutation and cell death (Robert K et al., 1996). Major cellular ROS included singlet oxygen $\left(\mathrm{O}_{2}\right)$, nitric oxide (NO), superoxide anion $\left(\mathrm{O}_{2}^{-}\right)$, hydrogen peroxide $\left(\mathrm{H}_{2} \mathrm{O}_{2}\right)$, hydroxyl radicals $\left(\mathrm{OH}^{\circ}\right)$, alkoxyl radicals (RO), peroxyl radicals $(\mathrm{ROO})$, and lipid peroxides $(\mathrm{LOOH})$. During periods of high oxidative stress or tissue injury, loss of ROS control can occur and ROS formation is favored (Beckman and Koppenol, 1996).

Several study have tried to identify the origin and characterize the AF associated with oxidative stress that results in a significant ROS increase. Michael et al., found that myofibrillar creatine kinase, an important controller of myocyte contractility, is highly sensitive to oxidative injury, and that increased oxidative stress and energetic impairment during AF could contribute to contractile dysfunction (Minm et al., 2001).
The atrial tissue subjected to rapid atrial pacing showed direct evidence of increased oxidative stress (increased 3-nitrotyrosine formation), and ascorbate was able to minimize this effect (Carnes et al., 2001). Although several events that contribute to oxidative stress are well-established consequences of sustained $A F$, underlying mechanisms and relationships between $A F$ and oxidative stress by high-throughput approaches are ill-defined. Recent developments in genome sciences have led to the development of DNA microarray technology, a tool of unprecedented power for the study of gene sequence, structure, and expression. Using cDNA microarrays, the expression of thousands of genes can be monitored simultaneously and expression patterns compared cDNA microarrays have been developed to reveal the gene expression patterns of up- or downregulated genes in response to various biological stimuli. The new technology allows automated imaging analysis and is well suited for the large-scale study of gene expression patterns in vitro and in vivo (Park et al., 2002).

In this study, we compared gene transcription profiles in postoperative AF patients with non-AF controls. Using a cDNA array approach, we assessed a causal relationship between oxidative stress and AF in accordance with the hypothesis that increased oxidative stress may underlie AF derived from postoperative oxidative stress and electrophysiological remodeling such as atrial pacing.

\section{Materials and Methods}

\section{Patients}

Atrial appendages were obtained as surgical specimens from patients undergoing cardiac surgery using procedures approved by Korea University Medical Center. All patients gave informed consents. Right artrial appendages were obtained from 26 patients in permanent $\mathrm{AF}$ ( $>1$ month at the time of surgery) undergoing the Maze procedure and mitral valve repair (mean age 50, range 25-68 years). Control data were obtained from the right appendages of 26 patients in normal sinus rhythm with no history of AF and undergoing cardiac surgery (mean age 53, 27-65 years). Surgeries were performed between January 2000 and July 2001. Table 1 details clinical characteristics of $\mathrm{AF}$ patients.

\section{Human cDNA microarray}

A human cDNA microarray was primarily derived from a commercially available master set of approximately 15,000 human verified sequences (Research Genetics, Inc., Huntsville, AL). The 15,000-human cDNA clone set was sorted for a list of genes (1,152 elements) 
Table 1. Baseline characterisitics of the patient with atrial fibrillation.

\begin{tabular}{|c|c|}
\hline Clinical characterisitics & Results \\
\hline Number of patient & 26 \\
\hline $\operatorname{Sex}(M / F)$ & $23 / 3$ \\
\hline \multicolumn{2}{|l|}{ Age (yr) } \\
\hline Mean & 50 \\
\hline Range & $25-68$ \\
\hline \multicolumn{2}{|l|}{ Symptoms of atrial fibrillation } \\
\hline Duration (yr) & 7.4 \\
\hline Episode (range) & $1 /$ day $-1 /$ mon \\
\hline Lasting (range) & $10 \mathrm{~min}-48 \mathrm{~h}$ \\
\hline $\begin{array}{l}\text { Previous external cardioversion } \\
\text { (All cases recurred) }\end{array}$ & $7 / 26$ \\
\hline \multicolumn{2}{|l|}{$\begin{array}{l}\text { Previous antiarrhythmic drugs } \\
\text { unsuccessful drugs }\end{array}$} \\
\hline Number & $3-4$ \\
\hline \multirow[t]{6}{*}{ Mean } & 2.8 \\
\hline & Amiodarone $(n=6)$ \\
\hline & Propafenone $(n=9)$ \\
\hline & Sotalol $(n=1)$ \\
\hline & Flecainide $(n=8)$ \\
\hline & Quinidine $(n=2)$ \\
\hline History of thromboembolism & $2 / 26$ \\
\hline \multicolumn{2}{|l|}{ Underlying heart disease } \\
\hline Hypertension & 6 \\
\hline Atrial septal defect & 1 \\
\hline Dilated cardiomyopathy & 1 \\
\hline
\end{tabular}

representing families such as differentiation, development, proliferation, transformation, cell-cycle progression, immune response, transcription and translation factors, oncogenes, and molecules involved in cell growth and maintenance. PCR-amplified cDNAs were spotted on nylon membranes. The general methodology of arraying is based on the procedures of DeRisi et al. (1996).

\section{CDNA radiolabeling}

Total RNAs prepared from cardiac tissues of patients with or without $A F$ were used to synthesize ${ }^{33} \mathrm{P}$. labeled cDNAs by reverse transcription. Briefly, 3-10 $\mu \mathrm{g}$ of RNA were labeled in a reverse transcription reaction containing $5 \mathrm{X}$ first-strand $\mathrm{PCR}$ buffer, $1 \mu \mathrm{g}$ of 24-mer poly dT primer, $4 \mu \mathrm{l}$ of $20 \mathrm{mM}$ each dNTP excluding dCTP, $4 \mu$ of $0.1 \mathrm{M}$ DTT, $40 \mathrm{U}$ of RNase inhibitor, $6 \mu$ of $3,000 \mathrm{Ci} / \mathrm{mmol} \alpha-{ }^{33} \mathrm{P}$ dCTP to a final volume of $40 \mu$ l. The mixture was heated at $65^{\circ} \mathrm{C}$ for $5 \mathrm{~min}$, followed by incubation at $42^{\circ} \mathrm{C}$ for $3 \mathrm{~min}$. Two $\mu$ (specific activity: $200,000 \mathrm{U} / \mathrm{ml}$ ) of Superscript II reverse transcriptase (Life Technologies, Inc., Rockville, MD) was then added and the samples were incubated for $30 \mathrm{~min}$ at $42^{\circ} \mathrm{C}$, followed by the addition of $2 \mu$ of Superscript II reverse transcriptase and another $30 \mathrm{~min}$ of incubation. Five $\mu \mathrm{l}$ of $0.5 \mathrm{M}$ EDTA was added to chelate divalent cations. After the addition of $10 \mu \mathrm{l}$ of $0.1 \mathrm{M} \mathrm{NaOH}$, the samples were incubated at $65^{\circ} \mathrm{C}$ for $30 \mathrm{~min}$ to hydrolyze the remaining RNA. Following the addition of $25 \mu$ of $1 \mathrm{M}$ Tris ( $\mathrm{pH}$ 8.0), the samples were purified using BioRad 6 purification columns (Hercules, CA). This resulted in $5 \times 10^{6}$ to $3 \times 10^{7} \mathrm{cpm}$ per reaction (Vawter et al., 2001).

\section{Hybridization \& scanning}

cDNA microarrays were pre-hybridized in hybridization buffer containing $4.0 \mathrm{ml}$ Microhyb (Research Genetics), $10 \mu \mathrm{l}$ of $10 \mathrm{mg} / \mathrm{ml}$ human Cot 1 DNA (Life Technologies), and $10 \mu \mathrm{l}$ of $8 \mathrm{mg} / \mathrm{ml}$ poly $\mathrm{dA}$ (Pharmacia, Peapack, NJ). Both Cot 1 and poly $d A$ were denatured at $95^{\circ} \mathrm{C}$ for $5 \mathrm{~min}$ prior to use. After $4 \mathrm{~h}$ of prehybridization at $42^{\circ} \mathrm{C}$, approximately $10^{7} \mathrm{cpm} / \mathrm{ml}$ of heat-denatured $\left(95^{\circ} \mathrm{C}, 5 \mathrm{~min}\right)$ probes were added and incubation continued for $17 \mathrm{~h}$ at $42^{\circ} \mathrm{C}$. Hybridized arrays were washed three times in $2 \times$ SSC and 0.1 $\%$ SDS for $15 \mathrm{~min}$ at room temperature. The microarrays were exposed to phosphoimager screens for 1-5 days, and the screens were then scanned in a FLA-8000 (Fuji Photo Film Co., Japan) at 50-um resolution (Vawter et al., 2001).

\section{Data analysis}

Microarray images were trimmed and rotated for further analysis using L-Processor (Fuji Photo Film Co., Japan). Gene expression of each microarray was captured by the intensity of each spot produced by radioactive isotopes. Pixels per spot were counted by Arrayguage (Fuji Photo Film Co., Japan) and exported to Microsoft Excel (Microsoft, Seattle, WA). The data were normalized with $Z$ transformation to obtain $Z$ scores by subtracting each average of gene intensity and dividing with each standard deviation. Z scores provide each of 2,304 spots (two sets of 1,152 genes) genes with the distance from the average intensity and were expressed in units of standard deviation. Thus, each $Z$ score provides flexibility to compare different sets of microarray experiments by adjusting differences in hybridization intensities. Gene expression difference as compared with untreated control cells was calculated by comparing $Z$ score differences ( $Z$ differences) among the same genes. This facilitates comparing each gene that had been up- or downregulated as compared with the control cells. $Z$ differences were calculated first by subtracting $\mathbf{Z}$ 
scores of the controls from each Z score of the samples. These differences were normalized again to distribute their position by subtracting the average $Z$ difference and dividing with the standard deviation of the $Z$ differences. These distributions represent the $Z$ ratio value and provide the efficiency for comparing each microarray experiment (Vawter et al., 2001). Scatter plots of intensity values were produced by Spotfire (Spotfire, Inc., Cambridge, MA) (Tanaka et al., 2000). Cluster analysis was performed on Ztransformed microarray data by using two programs available as shareware from Michael Eisen's labor- atory (http://rana.lbl.gov). Clustering of changes in gene expression was determined by using a public domain cluster based on pair-wise complete-linkage cluster analysis (Eisen et al., 1998).

\section{Western blot analysis}

Cardiac tissues were washed twice with ice-cold PBS, lysed in buffer $(20 \mathrm{mM}$ Tris/ $\mathrm{HCl}, 100 \mathrm{mM} \mathrm{NaCl}, 5 \mathrm{mM}$ $\mathrm{MgCl}_{2}, 1 \%$ NP- $40,0.5 \%$ sodium deoxycholate, 100 $\mu \mathrm{M}$ pepstatin, $100 \mu \mathrm{M}$ antipain, $100 \mu \mathrm{M}$ chymostatin, $10 \mathrm{~g} / \mathrm{ml}$ leupeptin, $0.5 \mathrm{mM}$ phenylmethylsulfonyl fluo-

A

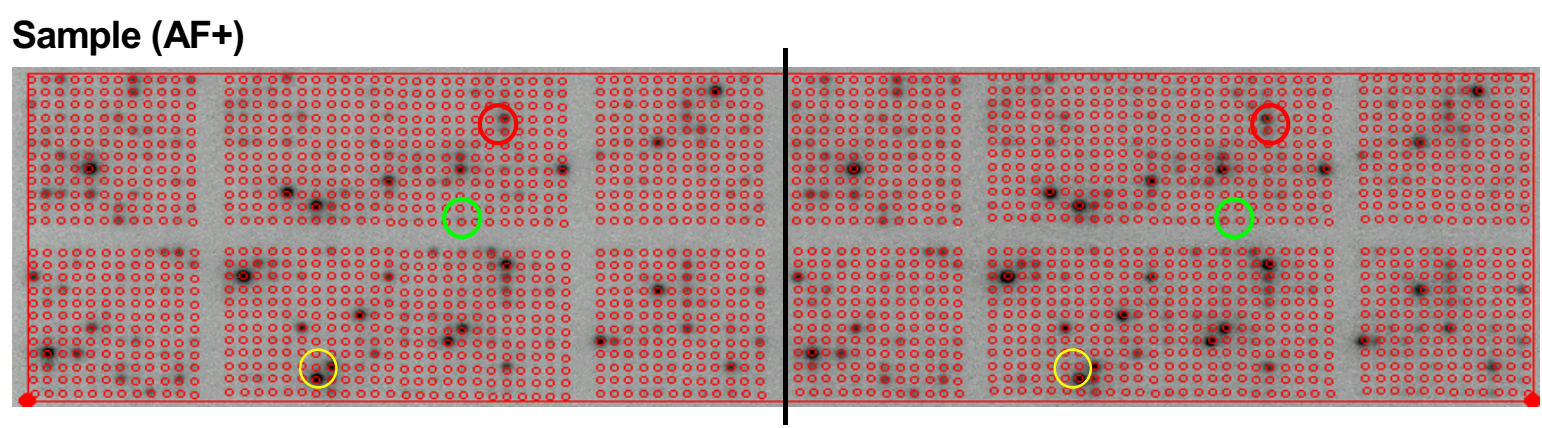

\section{Control (AF-)}

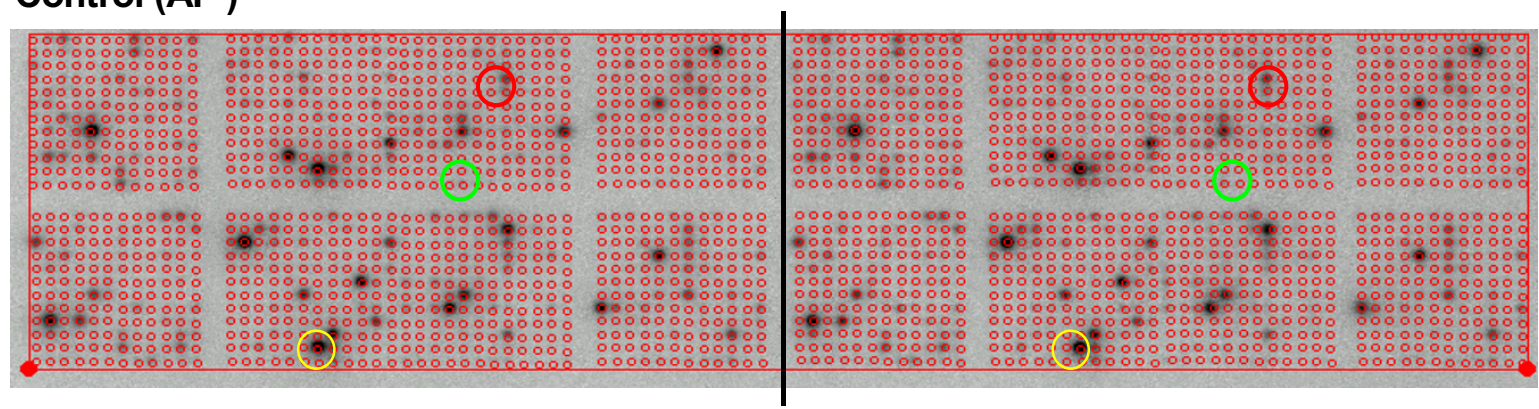

B

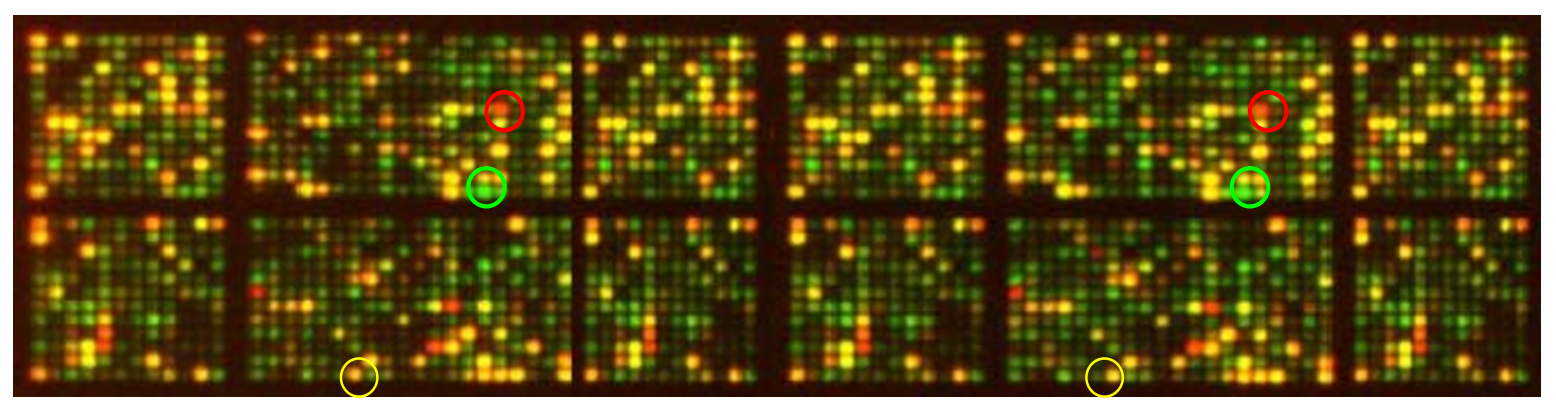

Figure 1. (A). Representative cDNA microarrays of two independent hybridization experiments comparing cDNAs generated from controls (down) or from atrial fibrillation (AF) patients (up). The cDNA microarray contained the two sets of 1,152 genes and printed in duplicate (as indicated by the line), and each duplicate is composed of eight individual subarrays. For example, two genes differentially expressed between controls and AF patients are marked by circles (red, monoamine oxidase B; green, glutathione peroxidase 1; yellow, GAPDH). (B) Superimposed image of primary images of controls and AF patients. 
ride, $5 \mu \mathrm{g} / \mathrm{ml}$ trypsin inhibitor, and $1 \mathrm{mM}$ benzamidine, $\mathrm{pH} 7.5$ ), and placed on ice for $15 \mathrm{~min}$. Protein concentration was determined by the Bradford assay using the Bio-Rad protein assay kit (Richmond, CA). Western analysis was performed according to the described method (Srivastava et al., 1999). Equal amounts of total cellular protein from tissue lysates of both groups were fractionated on a 10\% SDS-polyacrylamide gel. The proteins were transferred to a nitrocellulose membrane and incubated with 10\% nonfat dry milk in PBST (PBS, pH 7.4 with $0.2 \%$ Tween-20) for $1 \mathrm{~h}$ at room temperature. Polyclonal antibodies for tyrosinase-related protein 1, tyrosine 3-monooxygenase and heme oxygenase 2 were purchased from Santa Cruz Biotechnology, Inc. (Santa Cruz., CA). The bands of interest were visualized by chemiluminescence (ECL, Amersham Corp., Arlington Heights, IL).

\section{Results}

\section{Clinical assessment}

Clinical characteristics of AF patients are presented in Table 1. No age- or sex-related differences in AF and control patients were detected in any functional parameter measured (Spearman's correlation analysis).

\section{Gene expression profiles in AF patients}

Radioactive hybridization was visualized by phosphoimager technologies. The primary image, that is the results of primary capture by phosphoimager, is shown in Figure $1 \mathrm{~A}$. This particular array was printed in duplicate (as indicated by the line) and each duplicate was composed of eight individual subarrays. Visual inspection of the hybridization patterns readily identified a number of signals differentially expressed between normal and diseased tissue. Figure $1 \mathrm{~B}$ is a

Table 2. Up-requlated qene expression in riaht atrial appendage of AF evaluated by cDNA arrav.

\begin{tabular}{|c|c|c|c|c|}
\hline \multirow{2}{*}{ Gene name } & \multicolumn{2}{|c|}{ Z-value $^{1)}$} & \multirow{2}{*}{ Z-difference ${ }^{2)}$} & \multirow{2}{*}{ Z-ratio ${ }^{3)}$} \\
\hline & Control & AF & & \\
\hline Adenylate cyclase 9 & -4.98 & -0.41 & 4.57 & 5.81 \\
\hline Fatty acid binding protein 7 , brain & -4.60 & -0.31 & 4.3 & 5.45 \\
\hline Monoamine oxidase B & -3.72 & -0.05 & 3.66 & 4.65 \\
\hline Cyclin-dependent kinase 5 . regulatory subunit 1 (p35) & -3.78 & -0.13 & 3.65 & 4.63 \\
\hline Glycoprotein (transmembrane) $\mathrm{nmb}$ & -3.60 & -0.09 & 3.52 & 4.46 \\
\hline Nuclear receptor subfamily 3 , group $C$, member 1 & -3.88 & -0.53 & 3.35 & 4.26 \\
\hline Flavin containing monooxygenase 1 & -3.55 & -0.42 & 3.13 & 3.98 \\
\hline Synaptopodin & -3.03 & -0.07 & 2.96 & 3.76 \\
\hline Microtubule-associated protein 4 & -2.74 & 0.1 & 2.85 & 3.61 \\
\hline Amyloid beta (A4) precursor protein-binding, family $A$, member 3 (X11-like 2) & -3.06 & -0.6 & 2.80 & 3.56 \\
\hline General transcription factor $\mathrm{IIH}$, polypeptide 2 (44kD subunit) & -3.09 & -0.3 & 2.79 & 3.54 \\
\hline Protein kinase $\mathrm{C}$, mu & -2.95 & -0.19 & 2.75 & 3.50 \\
\hline Tyrosinase-related protein 1 & -3.02 & -0.33 & 2.69 & 3.41 \\
\hline Glutamate receptor, ionotropic, AMPA 2 & -2.82 & -0.19 & 2.62 & 3.33 \\
\hline Ubiquitin-conjugating enzyme E2D 2 (homologous to yeast UBC4/5) & -2.04 & 0.27 & 2.30 & 2.92 \\
\hline V-fos FBJ murine osteosarcoma viral oncogene homolog & -2.00 & 0.18 & 2.18 & 2.77 \\
\hline Gamma-aminobutyric acid (GABA) A receptor, pi & -2.01 & -0.04 & 1.98 & 2.51 \\
\hline Early growth response 2 (Krox-20 (Drosophila) homolog) & -2.29 & -0.33 & 1.96 & 2.49 \\
\hline Phosphoglycerate mutase 1 (brain) & -1.92 & 0.02 & 1.94 & 2.46 \\
\hline Gene near HD on $4 p 16.3$ with homology to hypothetical S. pombe gene & -2.11 & -0.18 & 1.93 & 2.44 \\
\hline
\end{tabular}

1) $Z$-value (gene1) $=\log _{10}$ [raw intensity (gene1)] $\log _{10}$ [mean raw intensity(all genes)] $/$ standard deviation $\log _{10}$ [raw intensity(all genes)], 2) Z-difference (gene1) $=Z_{(\text {gene1,array1) }}-Z_{(\text {gene1,array2), 3) } Z \text {-ratio(gene1) }}=Z_{\text {-difference }}$ (gene1) $_{1} / \operatorname{Sdev}_{(\text {Z-difference all genes) }}$ 
Table 3. Down-requlated gene expression in right atrial appendage of AF evaluated by cDNA array.

\begin{tabular}{|c|c|c|c|c|}
\hline \multirow{2}{*}{ Gene name } & \multicolumn{2}{|c|}{ Z-value ${ }^{1)}$} & \multirow{2}{*}{ Z-difference ${ }^{2)}$} & \multirow{2}{*}{ Z-ratio $^{3)}$} \\
\hline & Control & $\mathrm{AF}$ & & \\
\hline Human cadherin-associated protein-related (cap-r) mRNA, complete cds & -0.50 & -3.22 & -2.72 & -3.45 \\
\hline Actinin, alpha 4 & -0.60 & -2.83 & -2.23 & -2.83 \\
\hline Survival of motor neuron 1 , telomeric & -0.43 & -2.62 & -2.19 & -2.77 \\
\hline G protein-coupled receptor kinase 6 & -0.34 & -2.49 & -2.14 & -2.72 \\
\hline Megakaryocyte stimulating factor & 0.30 & -1.84 & -2.13 & -2.71 \\
\hline Potassium inwardly-rectifying channel, subfamily $\mathrm{J}$, member 8 & 0.08 & -1.98 & -2.06 & -2.61 \\
\hline RAB32, member RAS oncogene family & -0.42 & -2.46 & -2.03 & -2.58 \\
\hline KIAA0421 protein & 0.20 & -1.79 & -1.99 & -2.53 \\
\hline Dynein, cytoplasmic, intermediate polypeptide 1 & -1.04 & 2.98 & -1.95 & -2.47 \\
\hline Supervillin & -1.32 & -3.24 & -1.92 & -2.44 \\
\hline Neuronal pentraxin II & -0.67 & -2.48 & -1.81 & -2.29 \\
\hline DNA-damage-inducible transcript 3 & -0.56 & -2.32 & -1.76 & -2.24 \\
\hline Hsp70-interacting protein & -0.24 & -1.98 & -1.73 & -2.20 \\
\hline DKFZP564G2022 protein & -0.70 & -2.39 & -1.69 & -2.15 \\
\hline Heme oxygenase (decycling) 2 & 0.37 & -1.29 & -1.66 & -2.11 \\
\hline Homo sapiens clone 683 unknown mRNA, complete sequence & -0.67 & -2.34 & -1.67 & -2.11 \\
\hline Myosin phosphatase, target subunit 1 & -0.11 & -1.75 & -1.64 & -2.08 \\
\hline Synaptojanin 2 & 0.50 & -1.14 & -1.64 & -2.08 \\
\hline Histone acetyltransferase 1 & -0.23 & -1.86 & -1.63 & -2.06 \\
\hline Supertransperase & -0.03 & -1.66 & -1.63 & -2.06 \\
\hline
\end{tabular}

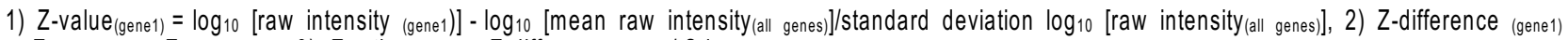

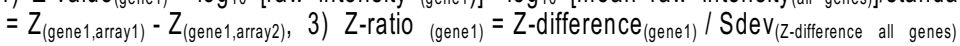

superimposed image in which red color represents upregulation and green represents downregulation in AF patients, and yellow represents genes of higher expression in AF and controls, such as housekeeping genes. Analysis of the median densitometric signal intensity revealed that 55 genes differed between the atrial tissue of AF patients and controls by a Z-ratio of 2 at a descriptive $P \leq 0.05$. We marked three genes: two genes differentialy expressed between AF and control tissues (red, monoamine oxidase B; green, glutathione peroxidase) and one gene similarly expressed between AF and controls (yellow, GAPDH). Using the cDNA expression array, we found that among 1,152 genes on the array membrane, $20 \%$ of total genes presented a quantifiable expression in right atrial appendages. Gene expression profiles of interest were significantly upregulated (30 genes) or downregulated (25 genes) in AF patients when compared with control patients. The top 20 prominently changed genes are listed in Table 2 and 3. Genes showing highly altered expression levels were aligned in the order of the magnitude of altered expression in AF patients'atrial appendages.

\section{Evaluation of ROS genes associated with AF}

Table 4 shows the gene expression pattern for differentially expressed genes with putative relevance to the oxidative stress. These pro-oxidative and antioxidative genes were up- and downregulated, respectively, more in AF tissues than in control tissues. Comparison rank analysis revealed that the expression of eight genes related to oxidative stress, including monoamine oxidase $\mathrm{B}$, flavin-containing monooxygenase 1 , tyrosinase-related protein 1 , tyrosine 3 monooxygenase, ubiquitin-specific protease 8 , NADPH oxidase, cytochrome $P 450$, and xantine oxidase increased by more than 1.04 of the Z-ratio and six 
Table 4. Gene's related to pro- and anti-oxidation in AF patient by cDNA array.

\begin{tabular}{|c|c|c|c|c|}
\hline \multirow{2}{*}{ Gene name } & \multicolumn{2}{|c|}{ Z-value ${ }^{1)}$} & \multirow{2}{*}{ Z-difference ${ }^{2)}$} & \multirow{2}{*}{ Z-ratio $^{3)}$} \\
\hline & Control & AF & & \\
\hline \multicolumn{5}{|l|}{ Pro-oxidant } \\
\hline Monoamine oxidase $B$ & -3.72 & -0.05 & 3.66 & 4.65 \\
\hline Flavin containing monooxygenase 1 & -3.55 & -0.42 & 3.13 & 3.98 \\
\hline Tyrosinase-related protein 1 & -3.02 & -0.33 & 2.69 & 3.41 \\
\hline Tyrosine 3-monooxygenase & -1.46 & 0.22 & 1.68 & 2.14 \\
\hline Ubiquitin specific protease 8 & -1.40 & -0.26 & 1.14 & 2.00 \\
\hline NADPH oxidase & -1.32 & -0.08 & 1.24 & 1.58 \\
\hline Cytochrome P 450 & -1.44 & -0.83 & 0.63 & 1.11 \\
\hline Xanthine oxidase & -0.76 & 0.13 & 0.82 & 1.04 \\
\hline \multicolumn{5}{|l|}{ Anti-oxidant } \\
\hline Glutathione peroxidase 1 & -0.08 & -1.65 & -1.58 & -2.00 \\
\hline Heme oxygenase (decycling) 2 & 0.37 & -1.29 & -1.66 & -2.11 \\
\hline Glutaredoxin (thioltansferrase) & -0.53 & -1.95 & -1.46 & -1.85 \\
\hline Glutathione reductase & -0.44 & -1.02 & -0.58 & -0.73 \\
\hline Superoxide dismutase & -0.11 & -0.07 & 0.04 & 0.05 \\
\hline Catalase & -0.82 & -0.75 & 0.07 & 0.09 \\
\hline
\end{tabular}

1) $Z$-value $\left(\right.$ gene1) $=\log _{10}\left[\right.$ raw intensity (gene1)] $-\log _{10}$ [mean raw intensity $($ all genes) $] /$ standard deviation $\log _{10}$ [raw intensity(all genes)], 2) Z-difference (gene1) $=Z_{(\text {gene1,array1) }}-Z_{(\text {gene1,array2) }}$, 3) Z-ratio(gene1) $=Z$-difference ${ }_{(\text {gene1) }} / \operatorname{Sdev}_{(\text {Z-difference all genes) }}$

genes related to antioxidant, including glutathione peroxidase 1, heme oxigenase 2, glutaredoxin, glutathione reductase, superoxide dismutase, and catalase, decreased by less than 0.09 of the Z-ratio in at least one of the five-time multiplicated experiments. Distribution of these oxidative genes was distinct, which suggests differential sensitivities of AF patients to these oxidative events.

Figure 2 is scatter plot for comparing the expression profiles of $A F$ and control patients. Expression profiles of atrial appendages in AF and control patients are shown as scatter plot of 1,152 genes from the microarray. Regression analysis of $Z$ scores from two independent samples of $\mathrm{AF}$ and control were performed and $Z$ scores of individual genes were plotted. $A$ zone represented the genes similarly expressed between $A F$ and controls in general. The $B\left(B^{\prime}\right)$ zone and $C\left(C^{\prime}\right)$ zones represented up- and downregulated genes respectively. That $B^{\prime}$ zone genes showing a much higher upregulation than $B$ zones genes is in contrast with that of $C^{\prime}$ zone which showed a greater downregulation than $C$ zone.

To obtain a molecular portrait of relationships between increased oxidative stress and pathological alterations associated with $\mathrm{AF}$, we used a hierarchical clustering algorithm to group genes on the basis of similar expression patterns (Eisen et al., 1998), and the data are presented in a matrix format (Figure 3 , 4). Each row of Figure 3 and 4 represents all hybridization results for a single DNA element of the array, and each column represents the expression levels for all genes in a single hybridization sample. The expression level of each gene was visualized in color, relative to its median expression level across all samples. Red represented expression greater than the mean, green represents expression less than the mean, and color intensity denotes the degree of deviation from the mean. Gray represented median expression level. Distinct samples representing similar gene patterns from control cells were aligned in adjacent rows. The cells included in this map were samples from right atrial appendages of $A F$ and control patients. Coordinately expressed genes were grouped into clusters, which we named on the basis of the cellular process in which component genes participated. The clustergram revealed that clusters of genes related to oxidative stress were up- and downregulated in AF patients, as compared to controls (Figure 3 and 4).

\section{Western blot analysis}

In parallel experiments, gene expression of atrial appendages was identified by Western blot, in which 


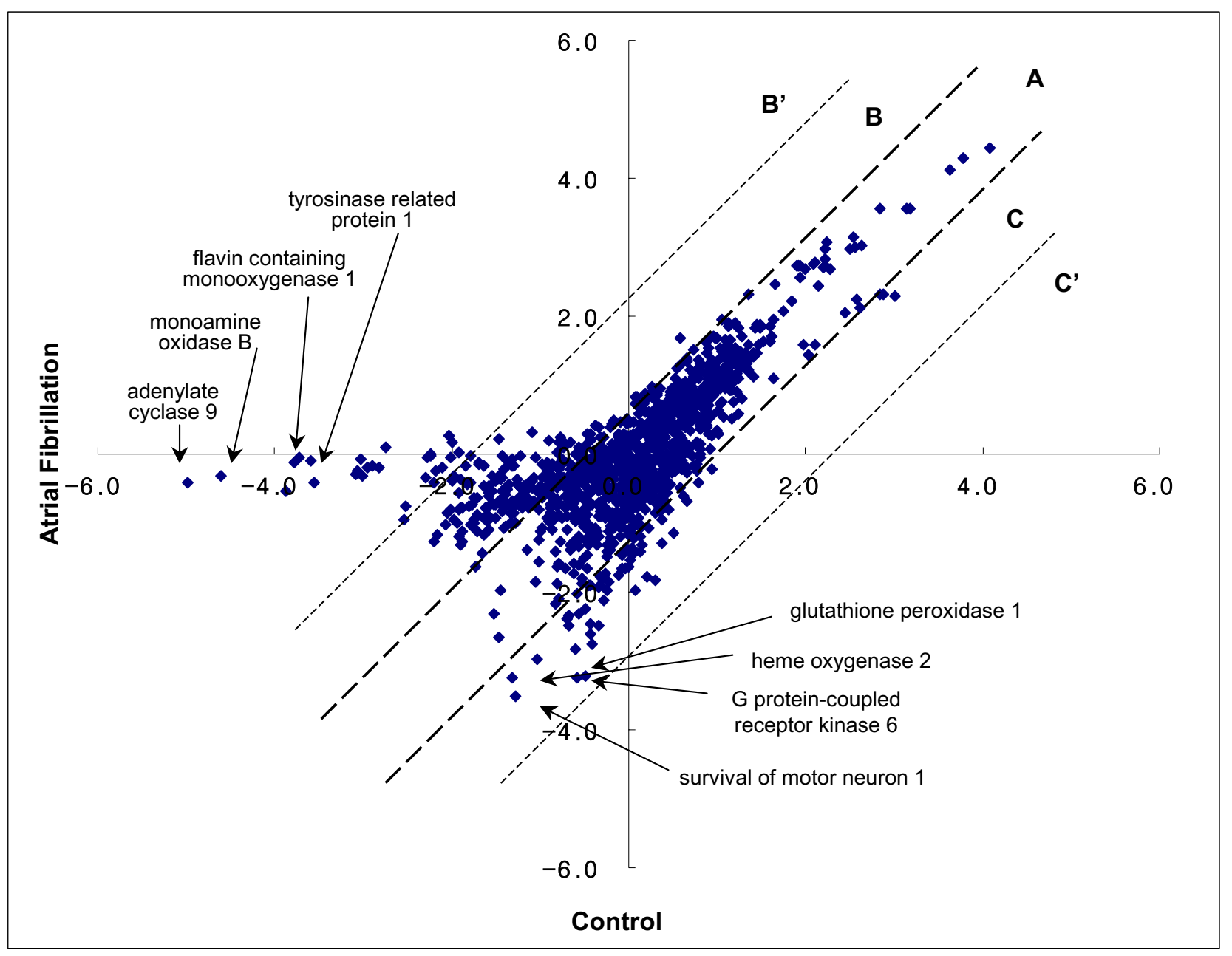

Figure 2. Scatter plot for comparison of expression profile between controls and AF patients. Expression profiles of atrial appendages in controls and AF patients are shown as bivariated scatter plot of 1,152 genes from the microarray. The values are corrected intensities relative to control, representing levels of expression for the CDNA elements of the microarrays.

the protein levels of tyrosinase-related protein 1, tyrosine 3-monooxygenase, and heme oxygenase 2 were assessed. Proteins extracted from right atrial appendages of AF and control patients were loaded in left and right lanes with duplicate and separated by SDS/ PAGE. As shown in Figure 5, the expression of tyrosinase-related protein 1 and tyrosine 3-monooxygenase levels were significantly increased in AF group compared to the control group. In contrast, the heme oxygenase 2 level was significantly decreased in the AF group compared to the control group.

\section{Discussion}

Most human disease is caused at least in part by ROS, and oxidative stress is thought to cause AF. In this study, we discovered novel evidence for pre- viously unknown patterns of gene expression events that related to oxidative stress of AF patients; e.g., upregulation of flavin containing monooxygenase 1 , monoamine oxidase $B$, ubiquitin-specific protease 8 , tyrosinase-related protein 1 , and tyrosine 3-monooxygenase, and down regulation of glutathione peroxidase 1 , and heme oxigenase 2 . To identify novel therapeutic targets for preventing human $A F$, this study compared gene expression profiles of atrial appendages in AF patients with those of control patients. cDNA microarray technology identifies differentially expressed genes with high sensitivity and fidelity and can correctly predict expression of corresponding proteins.

To evaluate the molecular portrait of oxidative effect, we selected a human cDNA microarray composed of 1,152 non-redundant human clones to examine a spectrum of genes affected by postoperative AF. 


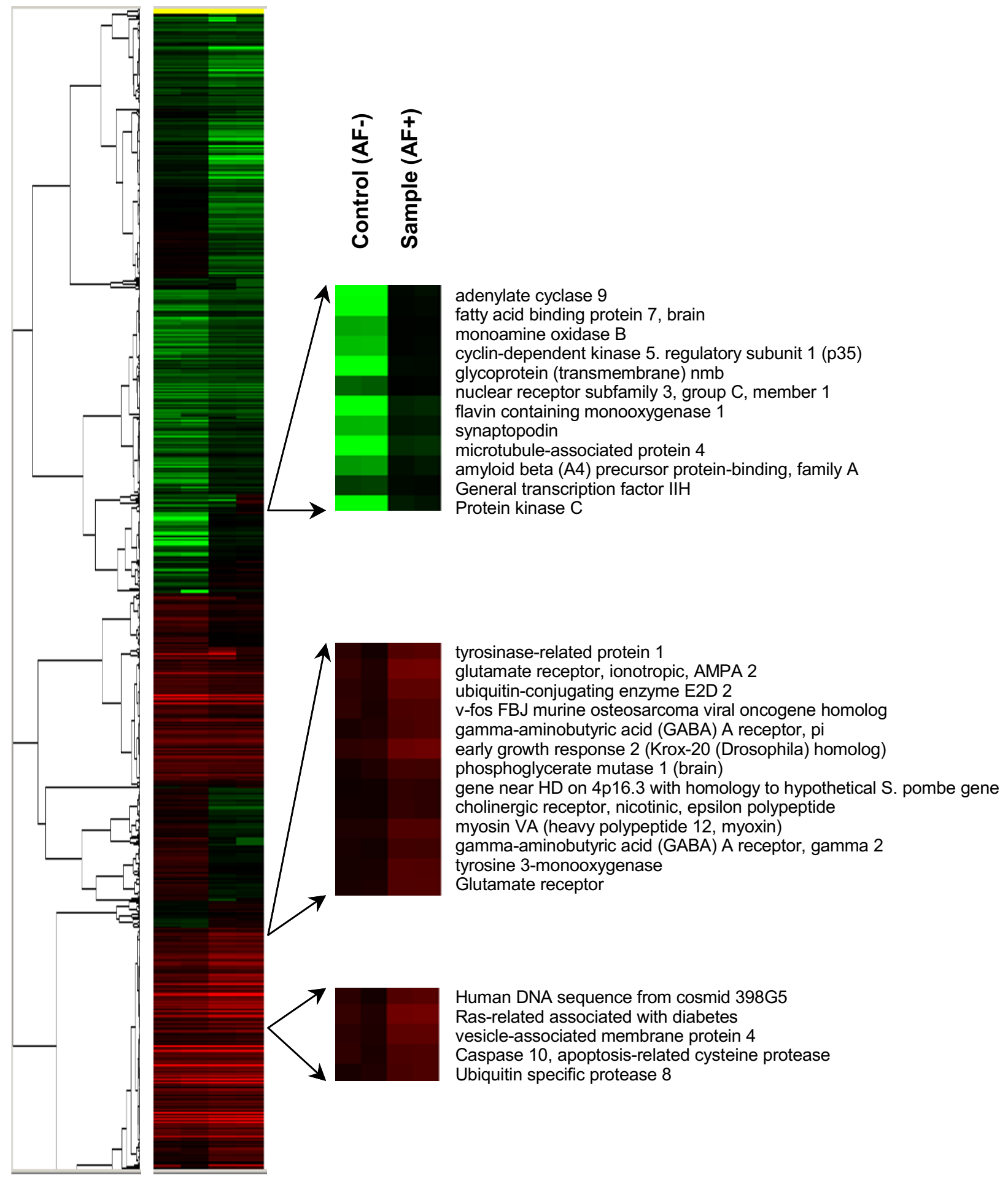

Figure 3. Clustergram of upregulated gene expression in AF. Microarray data from atrial tissue of controls and AF patients were combined and clustered. Cluster analysis was performed on Z-transformed microarray data using two separate programs available as shareware from Michael Eisen's lab. Each gene is represented by a single row of colored boxes; each experimental sample is represented by a single column. The entire clustered image is shown on the left. These clusters contain uncharacterized genes and genes not involved in these processes. 


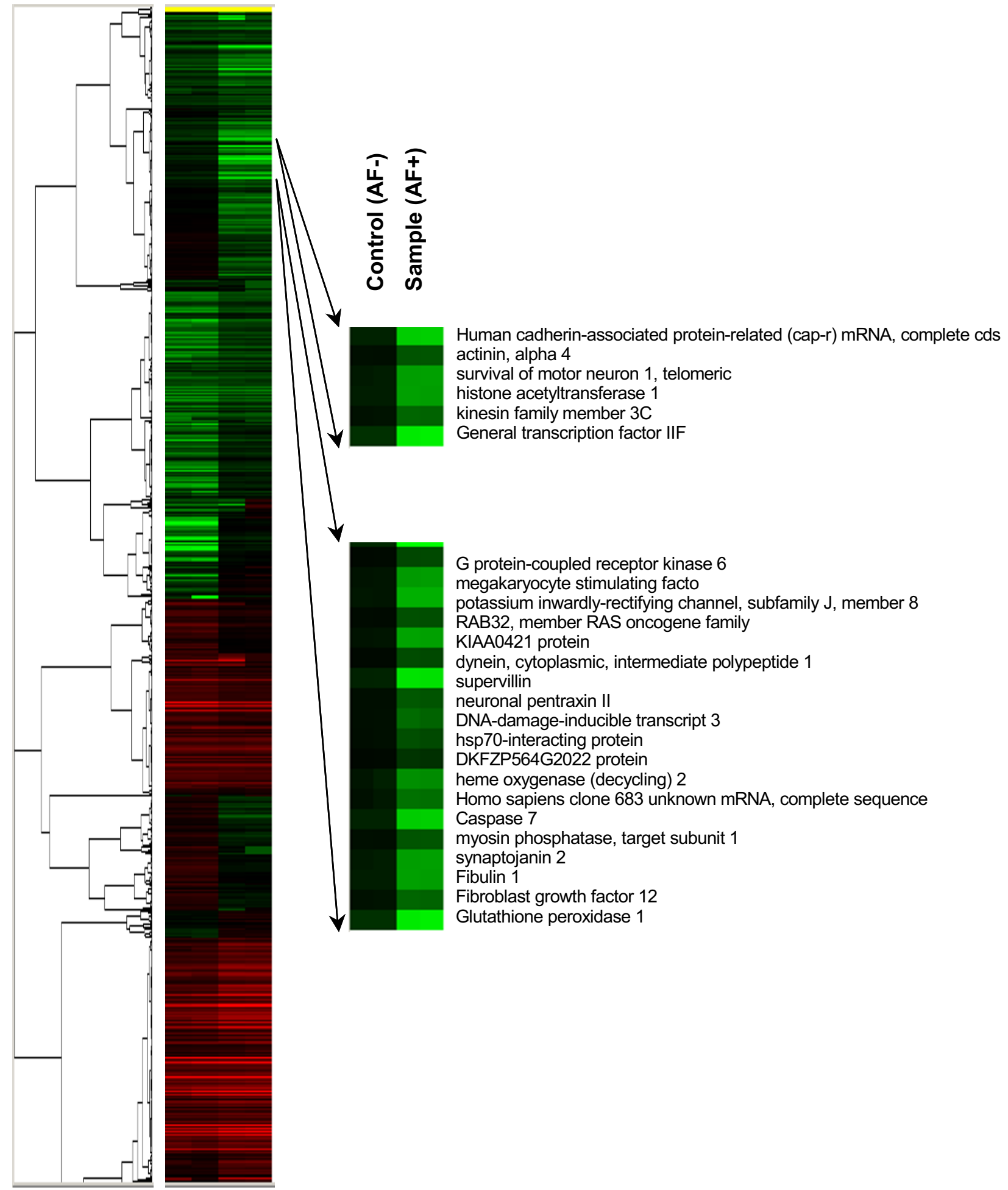

Figure 4. Clustergram of down-regulated gene expression in atrial fibrillation (AF). Microarray data from atrial tissue of controls and AF patients were combined and clustered. Cluster analysis was performed on Z-transformed microarray data using two separate programs available as share-ware from Michael Eisens lab. Each gene is represented by a single row of colored boxes; each experimental sample is represented by a single column. The entire clustered image is shown on the left. These clusters are contain uncharacterized genes and genes not involved in these processes. 


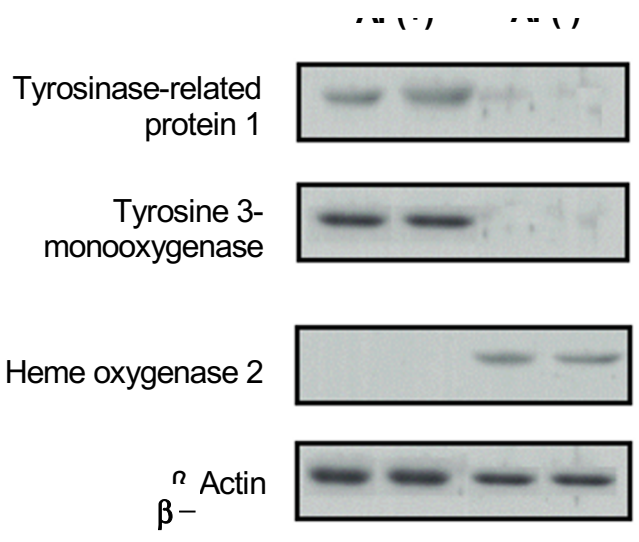

Figure 5. Representative Western blot analysis of tyrosine-related protein 1, tyrosine 3- monooxygenase, and heme oxygenase 2 in AF. Protein extracts from artrial appendages were loaded with duplicate samples run in the left and right lanes, respectively, and were separated by SDS/PAGE. The gels were transferred to nitrocellulose membrane and were immunoprobed for selected proteins using polyclonal antibodies. The same membrane was reprobed with the human $\beta$-actin probe as an internal loading control.

The microarray-based genomic survey is a highthroughput approach, that allowes parallel studies on expression patterns of thousands of genes (Konu et al., 2001). This technique can identify the correlation of oxidative stress and AF by performing comparisonrank analysis of genes expressed by the transcription of DNAs into RNAs. The amount of tissue was scant, containing $5 \times 10^{3}$ to $10 \times 10^{3}$ cells. To overcome technical problems posed by the limited amount of mRNA in such a low number of cells, we employed a new method of cDNA amplification. Higher concordance of hybridization signals and gene-specific PCR signals from house-keeping genes indicated that differences in hybridization signal intensity reflected variations of gene expression rather than variation of the cDNA microarray procedure.

$A F$, the most common sustained arrhythmia encountered in clinical practice, commonly occurs with rheumatic heart disease, particularly mitral stenosis. It also occurs with many other cardiac disorders, including coronary heart disease, congestive or hypertrophic cardiomyopathy, mitral valve prolapse, and mitral valve annular calcification. A number of potentially reversible, noncardiac factors are also associated with transient AF. The latter include hyperthyroidism, acute alcohol intoxification, cholinergic drugs, noncardiac surgery, and pulmonary conditions leading to hypoxemia. AF patients have marked atrial dilatation and atrial myocyte hypertrophy with increased interstitial fibrosis and fatty deposition (Mihm et al., 2001). AF is a frequent postoperative complication of cardiac surgery, with a reported incidence of $20 \%$ to
$50 \%$, increasing the risk of stroke. Patients undergoing coronary atrial bypass graft surgery have increased plasma lipid peroxidation and decreased cardiac glutathione levels following released of the cross clamp, and these changes persist for at least $24 \mathrm{~h}$ following cardiac surgery. Similarly, increased free-radical production in canine heart subjects lead to rapid ventricular pacing, and antioxidants can improve cardiac function in animals with pacinginduced failure (Carnes et al., 2001). In our experiments, we also reported that expression of oxidative genes-including monoamine oxidase $\mathrm{B}$, flavin-containing monooxygenase 1 , ubiquitin-specific protease 8 , tyrosinase-related protein 1 , and tyrosine 3-monooxygenase-increased in AF.

Oxidative stress is a biochemical modification of cells, tissues and lipids due to interactions with free radicals. Interactions can increase, decrease, or alter the function of specific proteins, depending on the degree and type of modification. Oxidative stress is also involved in pathological cardiovascular injury. Free radicals are highly unstable molecules that interact quickly and aggressively with other molecules in our bodies to create abnormal cells. They are capable of penetrating into and damaging the DNA of a cell so the cell will produce mutated cells that can replicate out of control. Free radicals are unstable because they have unpaired electrons in their molecular structure. This causes them to react almost instantly with any substance in their vicinity. Major cellular oxidant species include; 1) Nitric oxide, produced by a family of enzymes known as nitric oxide synthases. Production is increased in response to calcium overload; 2) superoxide, produced by xanthine oxidase and NADPH oxidase. Production is increased in response to Angiotensin II and by inflammatory responses; 3) $\mathrm{H}_{2} \mathrm{O}_{2}$; 4) Hydroxyl radical; and 5) Peroxynitrite, formed by the interaction of nitric oxide and superoxide. Peroxynitrite can covalently modify a variety of cellular lipids and proteins (Robert et al., 1996).

These reactive molecules can react with proteins, nucleic acids, lipids, and other molecules to alter their structure and produce tissue damage. The reactions play an important role in forming and disposing of ROS. Superoxide is formed in the cells by the action of enzymes such as cytochrome P450 reductase and xanthine oxidase. When stimulated by contact with bacteria, neutrophils exhibit a respiratory burst and produce superoxide in a reaction catalyzed by nicotine amide adenine dinucleotide phosphate (NADPH) oxidase. Superoxide dismutation converts $\mathrm{H}_{2} \mathrm{O}_{2}$ to water and oxygen and the rate of spontaneous superoxide dismutation is enhanced by the actions of superoxide dismutase and catalase, that are present in many types of cells. Myeloperoxidase uses $\mathrm{H}_{2} \mathrm{O}_{2}$ 
and halides to produce hypohalous acids. The selenium containing enzyme glutathione peroxidase will also act on reduced glutathione and $\mathrm{H}_{2} \mathrm{O}_{2}$ to produce oxidized glutathione disulfide and $\mathrm{H}_{2} \mathrm{O}$. $\mathrm{OH}^{\circ}$ can be formed from $\mathrm{H}_{2} \mathrm{O}_{2}$ in a nonenzymatic reaction catalyzed by $\mathrm{Fe}^{2+} . \mathrm{O}_{2}$ and $\mathrm{H}_{2} \mathrm{O}_{2}$ are the substrates in the iron-catalyzed Haber-Weiss reaction. NADPH plays a key role in supplying reducing equivalents in red blood cells and hepatocytes. NADPH also reduces glutathione disulfide (GSSG) to glutathione (GSH) catalyzed by glutathione reductase.

Chemical reactions capable of generating potential toxic ROS can be referred to as pro-oxidation, where reactions that dispose of theses species, scavenge them, suppress their formation, or oppose their actions are anti-oxidation. In normal cells, there is an appropriate pro-versus antioxidation balance. However, this balance can be shifted toward pro-oxidation. However, when production of oxygen species is greatly increased or when antioxidation levels are diminished. This state is called, oxidative stress, can result in serious cell damage if the stress is massive or prolonged. ROS are now thought to play an important role in many types of cellular injury, some of which can result in cell death. Indirect evidence supporting a role for ROS in generating cell injury is provided if administration of an enzyme such as superoxide dismutase or catalase is found to protect against cell injury in the situation under study (Allessie et al., 2001).

Cellular defenses against oxidants are antioxidant enzymes (such as catalase, superoxide dismutase, and peroxidases.) and antioxidant molecules (such as glutathione, vitamin $\mathrm{C}$, and vitamin E). Possible biochemical mechanisms for AF might be possible to calcium overload and neurohormonal activation. Calcium overload by oxidative stress can increase the production of nitric oxide (NO), and mitochondrial free radicals (Allessie et al., 2001; Van Wagoner, 2001). AF is associated with neurohormonal activation, frequently leading to increased production of superoxide.

In this study, we describe the upregulation of monoamine oxidase $B$, which enhances the release of $\mathrm{Ca}^{2+}$ from mitochondria at the mRNA and protein levels of human AF. Antioxidant ascorbate (vitamin C) can attenuate the electrical remodeling that accompanies rapid atrial pacing in an experimental model. Atrial tissue subjected to rapid atrial pacing showed direct evidence of increased oxidative stress (increased 3-nitrotyrosine formation), and ascorbate was able to minimize this effect. Further, supplemental ascorbate also helped to prevent tissue depletion of endogenous ascorbate (Carnes et al., 2001).

The most interesting results of the array study was upregulation of genes involved in facilitating oxidative stress and downregulation of genes involved in pro-

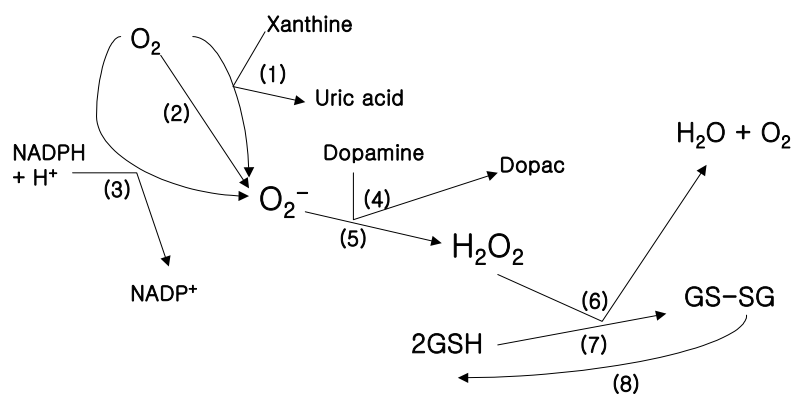

Figure 6. Pathway of reactive oxygen species (ROS) production and clearance. As shown in this pathway, the genes of xanthine oxidase, cytochrome P450, flavin-containing monooxygenase, monoamine oxidase $B$, and superoxide dismutase were related to ROS production, and the genes of catalase, glutathione peroxidase 1, and glutathione reductase were related to ROS clearance. In this study, gene expression of flavin-containing monooxygenase and monoamine oxidase $B$ were increased in the AF group compared to controls. In contrast, gene expression of glutathione peroxidase 1 was decreased in the AF group compared to controls. Abbreviations in this figures are as follows: $\mathrm{O}_{2}$, superoxide anion; $\mathrm{H}_{2} \mathrm{O}_{2}$, hydrogen peroxide; $\mathrm{GSH}$, glutathione; GSSG, glutathione disulfide; DOPAC, 3,4-dehydroxyphenylacetic acid; (1), xanthine oxidase; (2), cytochrome P450; (3), flavincontaining monooxygenase; (4), monoamine oxidase $B ;(5)$, superoxide dismutase; (6), catalase; (7), glutathione peroxidase $1 ;(8)$, glutathione reductase.

tecting against oxidative stress and oxidative damage repair (Figure 6). Here, we describe for the first time, to our knowledge, the upregulation of monoamine oxidase $B$ at the mRNA and protein levels of human AF. Monoamine oxidase B (Figure 6), a family of FAD-containing enzymes presents on the outer membrane of mitochondria, is involved in oxidative deamination of biologic amines and triggered mitochondrial damage leading to apoptosis or necrosis (Goudreau et al., 2002). Oxidative damage to mitochondrial DNA has been eported to occur when monoamine oxidase $B$ metabolizes tyramine (Hauptmann et al., 1996), and it has been demonstrated that $\mathrm{H}_{2} \mathrm{O}_{2}$ generated by monoamine oxidase $B$ activity enhances the release of $\mathrm{Ca}^{2+}$ and glutathione from mitochondria (Sandri et al., 1990). Moreover, ROS is known to stimulate a specific release of $\mathrm{Ca}^{2+}$ from mitochondria, and mitochondrial ROS production followed by enhanced $\mathrm{Ca}^{2+}$ cycling is known to cause apoptosis and necrosis (Richter et al., 1995). Ubiquitinspecific protease 8 is involved in oxidating dihydroxyindol and may regulate or influence the type of melanin synthesized. In Figure 6 (3), flavincontaining monooxygenase 1 is involved in the oxidative metabolism of a variety of xenobiotics such as drugs and pesticides. Other genes upregulated in AF patients were tyrosine 3-monooxygenase and tyrosinase-related protein 1, which are known to promote oxidative damage. As shown in Figure 6 (7), glutathione per- 
oxidase is a family of antioxidant enzymes that reduce $\mathrm{H}_{2} \mathrm{O}_{2}$ by the oxidation of reduced glutathione and protect against the toxic effects of oxidants generated within cells (Comhair et al., 2001). Glutathione peroxidase downregulation might be crucial to explaining ROS function in AF. Heme oxygenase 2 , an essential enzyme in heme catabolism, cleaves heme to form biliverdin, which is subsequently converted to bilirubin by biliverdin reductase, and carbon monoxide, a putative neurotransmitter. Bilirubin, a potent antioxidant, has been suggested to have cytoprotective properties (Lash et al., 2003). The present observation of the induction of genes involved in facilitating oxidation provides additional evidence of an important role for oxidative stress. In normal conditions a balance exists between free radical production and antioxidant/repair mechanisms, so that tissue injury is not promoted. The observed induction of several genes, and especially those of oxidative enzymes, is undoubtedly a result of the promotion of cell oxidation. In fact, oxidative stress conditions were shown to enhance the expression of several oxidant enzymes, that shift to pro-oxidation status in ROS metabolism. Based on this explanation, AF can be predisposed by altering the concentration of enzymes related to ROS metabolism and by shifting free radical balance to pro-oxidation instead of antioxidation.

We tested the hypothesis that oxidative stress could be related to human AF. This study provides novel evidences that gene expression for pro-oxidative stress occurs in atrial appendages during AF, and that a balance of pro-oxidation and anti-oxidation represents an important pathological mechanism in this arrhythmia. In summary, this study shows that the highly altered gene expression pattern related to oxidative stress may be an important event in the pathogenesis of $A F$, and that a variety of following studies are recommended to evaluate novel and more effective treatment strategies such as antioxidant therapy.

\section{Acknowledgement}

This work was supported by the Korean Health 21 R\&D Project of the Ministry of Health and Welfare (Grant\# HMP00-GN-01-002), the National Nuclear R\&D Program of the Ministry of Science and Technology, and the Korea Science and Engineering Foundation (Grant\# R01-2001-000-00212-0[2002]).

\section{References}

Allessie MA, Boyden PA, Camm AJ, Kleber AG, Lab MJ, Legato MJ, Rosen MR, Schwartz PJ, Spooner PM, Van Wagoner DR, Waldo AL. Pathophysiology and prevention of atrial fibrillation. Circulation 2001;103:769-77
Beckman JS, Koppenol WH. Nitric oxide, superoxide, and peroxynitrite: the good, the bad, and ugly. Am J Physiol 1996;271:C1424-37

Carnes CA, Chung, MK, Nakayama T, Nakayama H, Baliga RS, Piao S, Kanderian A, Pavia S, Hamlin RL, McCarthy PM, Bauer JA, Van Wagoner DR. Ascorbate attenuates atrial pacing-induced peroxynitrite formation and electrical remodeling and decreases the incidence of postoperative atrial fibrillation. Circ Res 2001;89:E32-8

Comhair SA, Bhathena PR, Farver C, Thunnissen FB, Erzurum SC. Extracellular glutathione peroxidase induction in asthmatic lungs: evidence for redox regulation of expression in human airway epithelial cells. FASEB J 2001;15:70-8

Eisen MB, Spellman PT, Brown PO, Botstein D. Cluster analysis and display of genome-wide expression patterns. Proc Natl Acad Sci USA 1998;95:14863-68

Goldfarb AH. Nutritional antioxidants as therapeutic and preventive modalities in exercise-induced muscle damage. Can J Appl Physiol 1999;24:249-66

Goudreau JL, Maraganore DM, Farrer MJ, Lesnick TG, Singleton $A B$, Bower JH, Hardy JA, Rocca WA. Case-Control study of dopamine transporter-1, monoamine oxidase-B, and catechol-O-methyl transferase polymorphisms in Parkinson's disease. Mov Disord 2002;17:1305-11

Hauptmann N, Grimsby J, Shih JC, Cadenas E. The metabolism of tyramine by monoamine oxidase $A / B$ causes oxidative damage to mitochondrial DNA. Arch Biochem Biophys 1996;335:295-304

Konu, O, Kane JK, Barrett T, Vawter MP, Chang R, Ma JZ, Donovan DM, Sharp B, Becker KG, Li MD. Region-specific transcriptional response to chronic nicotine in rat brain. Brain Res 2001;909:194-203

Kopecky SL, Gersh BJ. Dilated cardiomyopathy and myocarditis: natural history, etiology, clinical manifestations, and management. Curr Probl Cardiol 1987;12:569-647

Lash GE, McLaughlin BE, MacDonald-Goodfellow SK, Smith GN, Brien JF, Marks GS, Nakatsu K, Graham CH. Relationship between tissue damage and heme oxygenase expression in chorionic villi of term human placenta. Am J Physiol Heart Circ Physiol 2003;284:H160-7

Mihm MJ, Yu F, Carnes CA, Reiser PJ, McCarthy PM, Van Wagoner DR, Bauer JA. Impaired myofibrillar energetics and oxidative injury during human atrial fibrillation. Circulation 2001;104:174-80

Park GH, Choe J, Choo H-J, Park YG, Sohn JW, Kim MK. Genome-wide expression profiling of 8-chloroadenosine- and 8-chloro-cAMP-treated human neuroblastoma cells using radioactive human cDNA microarray. Exp Mol Med 2002; $34: 84-193$

Prystowsky, EN, Benson DW, Jr., Fuster V, Hart RG, Kay GN, Myerburg RJ, Naccarelli GV, Wyse DG. Management of patients with atrial fibrillation. A Statement for Healthcare Professionals. From the Subcommittee on Electrocardiography and Electrophysiology, American Heart Association. Circulation 1996;93:1262-77

Richter C, Gogvadze V, Laffranchi R, Schlapbach R, 
Schweizer M, Suter M, Walter P, Yaffee M. Oxidants in mitochondria: from physiology to diseases. Biochim Biophys Acta 1995;1271:67-74

Sandri G, Panfili E, Ernster L. Hydrogen peroxide production by monoamine oxidase in isolated rat-brain mitochondria: its effect on glutathione levels and $\mathrm{Ca} 2+$ efflux. Biochim Biophys Acta $1990 ; 1035: 300-5$

Srivastava RK, Srivastava AR, Seth P, Agrawal S, ChoChung YS. Growth arrest and induction of apoptosis in breast cancer cells by antisense depletion of protein kinase A-R1 alpha subunit: p53-independent mechanism of action. Mol Cell Biochem 1999;195:25-36
Tanaka TS, Jaradat SA, Lim M, Kargul GJ, Wang X Grahovac MJ, Pantano S, Sano Y, Piao Y, Nagaraja R. Genome-wide expression profiling of mid-gestation placenta and embryo using a 15,000 mouse developmental cDNA microarray. Proc Natl Acad Sci USA 2000;97:9127-32

Van Wagoner DR. Redox modulation of cardiac electrical activity. J Cardiovasc Electrophysiol 2001;12:183-4

Vawter MP, Barrett T, Cheadle C, Sokolov BP, Wood WH 3rd, Donovan DM, Webster M, Freed WJ, Becker KG. Application of cDNA microarrays to examine gene expression differences in schizophrenia. Brain Res Bull 2001;55:641-50 